\title{
Enhancing the Effectiveness of Intelligent Tutoring Systems Using Adaptation and Cognitive Diagnosis Modeling
}

\author{
Akrivi KROUSKA $^{\text {a,1 }}$, Christos TROUSSAS ${ }^{\text {a }}$, Filippos GIANNAKAS a , Cleo \\ SGOUROPOULOU ${ }^{a}$ and Ioannis VOYIATZIS ${ }^{a}$ \\ ${ }^{a}$ Department of Informatics and Computer Engineering University of West Attica, \\ Greece
}

\begin{abstract}
This paper presents a novel framework for developing educational hypermedia systems incorporating adaptation techniques and tailored feedback. In particular, the adaptation techniques refers to the content presentation; where the system hides/displays information according to students' knowledge level and learning goals, and to the navigation design; where the system proposes the learning path that is better to be followed based on their profile. Finally, the framework embodies a diagnostic model that analyzes the students' misconceptions and provides tailored feedback and advices on bridging students' knowledge gap. This framework aims to enhance the effectiveness of learning process, increasing student engagement through the adaptive content and navigation and improving student performance through the tailored feedback.
\end{abstract}

Keywords. Adaptive content, Adaptive navigation, Buggy model, Personalized learning, Tailored feedback

\section{Introduction}

Adaptive hypermedia increases the functionality of hypermedia by tailoring their presentation to each individual, and thus, providing an alternative to the traditional "onesize-fits-all" approach applied to the development of hypermedia systems [1]. Adaptive educational hypermedia systems construct a dynamic students' model based on their goals, preferences and knowledge, which is updated according to students' interactions with the system $[1,2]$. Hence, the systems adapt the presentation of their content and the navigation interface to each student's needs $[1,3]$.

An educational system focuses on a certain piece of knowledge, namely a course. The students' goal in an educational system is to learn the whole content provided or a significant part of it. The use of hypermedia helps students learn better. In particular, adapting the hypermedia to students' characteristics can improve students' performance and boost their engagement in learning process [4]. The main student characteristic that adaptive hypermedia systems use is student knowledge; since, the level of knowledge varies from one student to other, as well as each student has his/her own pace of learning [5]. Student knowledge emerged from the mistakes made during the assessment process. However, identifying why the student makes these mistakes can further help the student

\footnotetext{
${ }^{1}$ Corresponding Author, E-mail: akrouska@uniwa.gr.
} 
improve his/her cognitive state [6,7]. The diagnosis of student misconception is used for providing tailored feedback and advice with the intention of bridging the knowledge gap [8].

To this direction, this paper introduces a novel framework for developing effective educational hypermedia systems through the incorporation of adaptation techniques and cognitive diagnosis model. This framework can provide a new learning experience to students increasing student engagement and improving learning outcomes.

\section{Adapting content presentation and system navigation based on student knowledge level}

Adapting learning environments is crucial for confronting the differences between the students in their needs and preferences [9]. Users with different goals and knowledge may be interested in different pieces of information presented on a hypermedia page and may use different links for navigation [10]. Adaptive hypermedia systems include two basic areas for customizing their operations according to each student's individual needs: adaptive presentation and adaptive navigation.

Adaptive presentation refers to the function of adapting the content of a page based on student characteristics, such as knowledge level, learning goals and others [11]. In particular, it determines what information should be presented and how it should be organized and displayed. For example, the system should provide more detailed and advanced material to expert students, while it should provide more explanations and examples to novice students. The techniques used include the conditional text, stretch text, page variants, fragment variants and frame variants.

Adaptive navigation supports students in the learning environment by adapting the appearance and structure of user interface [12]. Hence, systems can support users in their navigation by limiting browsing space, suggesting most relevant links to follow, or providing adaptive comments to visible links, according to their profile. With this adaptation, students' learning experience and outcomes are expected to be improved. The adaptive navigation techniques used include the direct guidance, adaptive ordering, hiding and adaptive annotation.

\section{Providing tailored feedback based on student misconceptions}

Most of the tutoring systems consider only the number of mistakes a student makes in order to evaluate his/her knowledge. However, understanding why the student makes these mistakes is of major importance, since incomplete learning and forgetting content can be detected and faced using corresponding teaching strategies $[10,6]$. Thus, an enriched model of student cognitive state, including his/her misconception, is a prerequisite for guiding him/her properly in learning/training process [13].

To accomplish this goal, a diagnostic model for student misconception that also takes into consideration pedagogical aspects, needs to be developed. A solution includes the adoption of the Repair Theory introduced by [14]. According to this theory, every bug is related to a series of operations that generate an incomplete procedure and to a series of operations that represent the repair of the procedure so that it can proceed [15]. These two parts are independent; thus, the kind of repair attempted depends only on the procedure and its current impasse, not on how the incomplete procedure was derived. 
In view of the above, the diagnostic module needs to build a large repository of predefined bugs that is used for inserting the corresponding misconception to student model. This repository stores for each incorrect answer of test items, the kind of misconception concerned and the material referred to. Hence, every time students make mistakes, the system updates their profile and advices them which actions to be followed, e.g. the section of the lesson that needs to be studied more carefully, in order to "repair" their misconception ${ }^{2}$.

\section{A framework of adaptive tutoring systems incorporating a diagnostic model of student misconceptions}

This paper proposes a framework for developing an adaptive educational hypermedia system that facilitates the learning process and improves learning outcomes. The architecture of the system is based on the elements of intelligent tutoring systems. As such, it includes four modules, namely student, domain, tutor and interface module.

Regarding the student module, every time students give a test, the system records their mistaking behavior and updates properly their student profile, namely their knowledge level and misconceptions detected. The domain module stores data about the course content, assessment items, teaching strategies and buggy rules, as well as the relationship between them. The domain module feeds with data both the student module for building student profile and the tutor module for the adaptation process. The tutor module includes the adaptation techniques incorporated into the system for tailoring user interface and navigation, and the diagnostic model used for providing personalized feedback. To accomplish this, the tutor module combines the information emerged from student and domain module. Finally, the interface module delivers to students adaptive content regarding its presentation and navigation design. Furthermore, it provides tailored feedback to students according to their misconceptions in order to bridge the knowledge gap and improve learning outcomes.

The novelty of this framework is the adaptation techniques used and the diagnostic model applied. In particular, the proposed approach for the adaptive presentation is this of stretchtext, since it covers better the students' needs and system's goals for learning. Using the stretchtext approach, the system hides the expert knowledge from novice students and provides them to advanced students who need expertise. On the other hand, it provides additional examples and further explanations to novice students for enhancing the acquisition of knowledge. Regarding the adaptive navigation, the system incorporates the direct guidance and adaptive annotation. Using the direct guidance, when students $\log$ in, the system leads them to the lesson they need to study based on their progress in their previous visit. According to adaptive annotation approach, the system uses explanation icons next to lessons' links indicating the students' progress and thus, suggesting them an optional learning path to follow. It should be noted that students have the capability to study any lesson they want without the system restricting it to them. Finally, the system provides tailored feedback to students every time they give a test. The diagnostic model used for this purpose provides a mechanism for explaining the reason why a student makes a mistake, and not only identifies the mistakes. To this direction, a repository of buggy rules is constructed where each incorrect answer of a test item corresponds to a certain misconception of a lesson content. The buggy rules are

\footnotetext{
${ }^{2}$ http://tecfaetu.unige.ch/staf/staf-d/krige/staf11/buggy.html
} 
combined with the teaching strategies defined based on the learning goals that need to be achieved, providing useful advices to students on the learning path that can lead them to improve their knowledge.

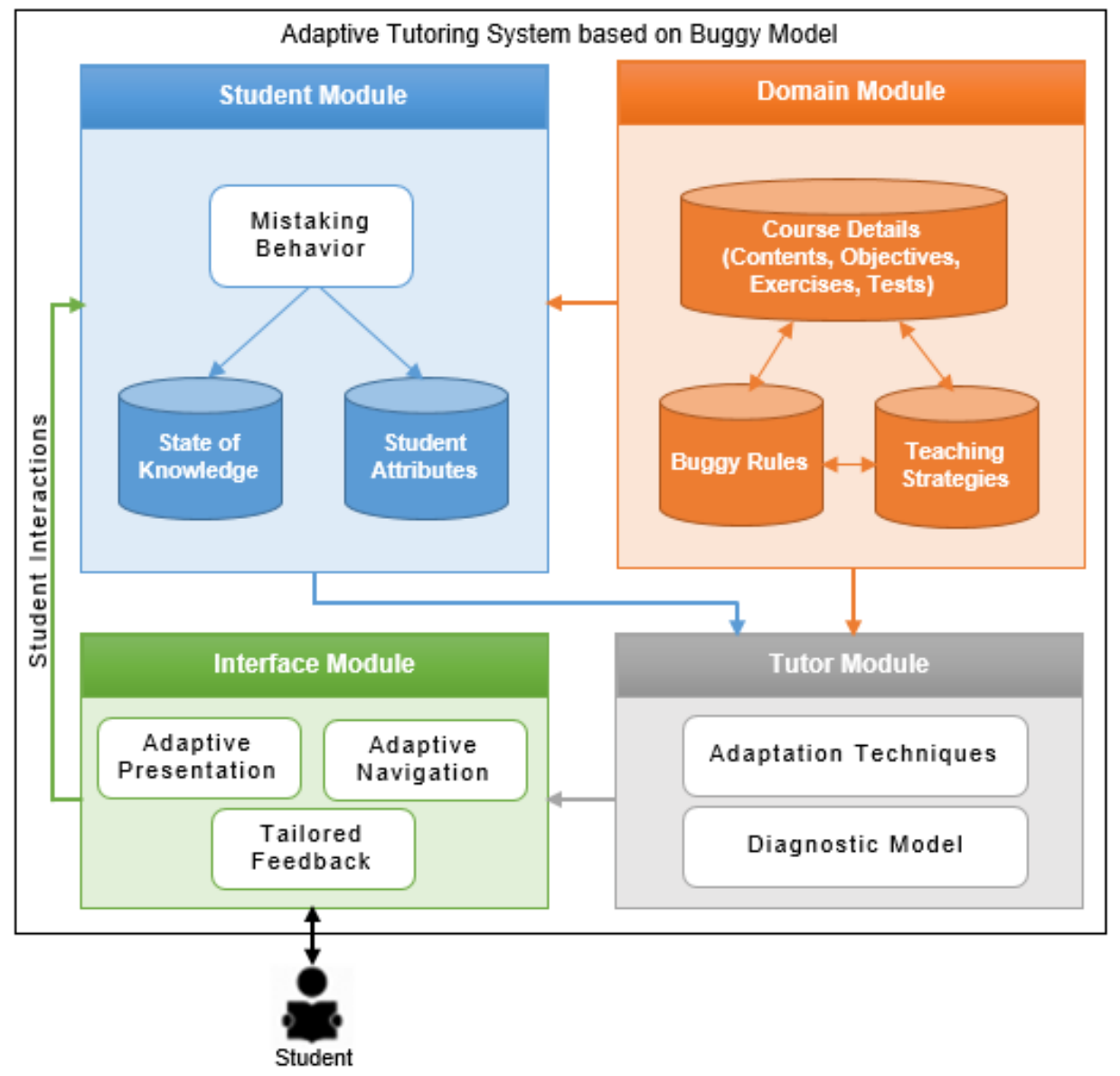

Figure 1. Architecture of proposed framework.

\section{Conclusions}

Adaptive educational hypermedia systems aims to guide students in their learning process in a tailored way, providing individualized learning instead of the traditional "one-size-fits-all" approach. To achieve their objective, they incorporate adaptation techniques and teaching strategies in order to deliver material to students based on their profile, needs, preferences and learning goals. The majority of tutoring systems consider students' knowledge level as the main characteristic for personalization identifying only the mistakes the students make. However, the diagnosis of the reason why a student make a mistake is of major importance for guiding properly in order to bridge knowledge gap.

To this direction, this paper introduces a novel framework for enhancing the effectiveness of tutoring systems. In particular, it incorporates adaptation techniques, 
namely adaptive presentation and navigation, and a cognitive diagnosis model based on student misconceptions. This framework is expected to boost student engagement and improve learning outcomes.

\section{References}

[1] P. Brusilovsky, Adaptive educational hypermedia, In International PEG Conference 10 (2001), 8-12.

[2] A. Krouska, C. Troussas and C. Sgouropoulou, A Personalized Brain-Based Quiz Game for Improving Students' Cognitive Functions, in Brain Function Assessment in Learning. BFAL 2020. Lecture Notes in Computer Science 12462 (2020), Cham, Springer.

[3] A. Krouska and M. Virvou, An Enhanced Genetic Algorithm for Heterogeneous Group Formation based on Multi-Characteristics in Social Networking-based Learning, IEEE Transactions on Learning Technologies 13(3) (2020), 465-476.

[4] C. Troussas, A. Krouska and C. Sgouropoulou, Collaboration and fuzzy-modeled personalization for mobile game-based learning in higher education, Computers \& Education 144 (2020), 103698.

[5] A. Krouska, C. Troussas and M. Virvou, Computerized Adaptive Assessment Using Accumulative Learning Activities Based on Revised Bloom's Taxonomy, In Joint Conference on Knowledge-Based Software Engineering (2018), 252-258.

[6] A. Krouska, C. Troussas and C. Sgouropoulou, Fuzzy logic for refining the evaluation of learners' performance in online engineering education, European Journal of Engineering Research and Science 4(6) (2019), 50-56.

[7] N. T. Heffernan, K. R. Koedinger and L. Razzaq, Expanding the model-tracing architecture: A 3rd generation intelligent tutor for algebra symbolization, International Journal of Artificial Intelligence in Education 18(2) (2008), 153-178.

[8] C. Troussas, A. Krouska and C. Sgouropoulou, Improving Learner-Computer Interaction through Intelligent Learning Material Delivery Using Instructional Design Modeling, Entropy 23(6) (2021), 668.

[9] C. Troussas, A. Krouska, C. Sgouropoulou and I. Voyiatzis, Ensemble Learning Using Fuzzy Weights to Improve Learning Style Identification for Adapted Instructional Routines, Entropy 22(7) (2020), 735.

[10] C. Troussas, A. Krouska, M. Virvou and E. Sougela, Using Hierarchical Modeling of Thinking Skills to Lead Students to Higher Order Cognition and Enhance Social E-Learning, In 2018 9th International Conference on Information, Intelligence, Systems and Applications (IISA) (2018), 1-5.

[11] A. Elmabaredy, E. Elkholy and A. Tolba, Web-based adaptive presentation techniques to enhance learning outcomes in higher education, Research and Practice in Technology Enhanced Learning 15(1) (2020), 1-18.

[12] A. Hamzah and K. Irianto, Adaptive Navigation Support: A Literature Review of Its Application and Implication in Learning Process, International Journal of Emerging Technologies in Learning (iJET) 14(16) (2019), 181-187. 
[13] C. Troussas, A. Krouska and M. Virvou, "sing a Multi Module Model for Learning Analytics to Predict Learners' Cognitive States and Provide Tailored Learning Pathways and Assessment, in Machine Learning Paradigms. Intelligent Systems Reference Library 158 (2020), Cham, Springer.

[14]J. Brown and K. VanLehn, Repair Theory: A Generative Theory of Bugs in Procedural Skills, Cogn. Sci. 4 (1980), 379-426.

[15] J. S. Brown and R. R. Burton, Diagnostic models for procedural bugs in basic mathematical skills, Cogn. Sci. 2(2) (1978), 155-192. 